\title{
IUFOST2006/499 \\ Using low-intensity ultrasound to investigate the effect of ingredi- ents and processing on the properties of breadmaking doughs
}

\author{
M. Scanlon ${ }^{\mathrm{a}}$, J. Page ${ }^{\mathrm{b}}$, H. Elmehdi ${ }^{\mathrm{c}}$, G. Bellido ${ }^{\mathrm{a}}$ and K. Mehta ${ }^{\mathrm{a}}$ \\ ${ }^{a}$ University of Manitoba, Department of Food Science, 250 Ellis Building, R3T2N2 Winnipeg, Canada \\ ${ }^{\mathrm{b}}$ University of Manitoba, Department of Physics \& Astronomy, R3T 2N2 Winnipeg, Canada \\ ${ }^{\mathrm{c}}$ University of Sharjah, Division of Physics, 34234 Sharjah, United Arab Emirates \\ scanlon@cc.umanitoba.ca
}

\begin{abstract}
There is a real need in modern breadmaking processes for tools that can non-invasively monitor the effect of processing and ingredients on the rheological properties of dough systems in order to better predict the cellular structure of the resulting loaf of bread. In this talk, we show that low-intensity ultrasound is a powerful tool for meeting this need. Low-frequency ultrasound $(\tilde{5} 0 \mathrm{kHz})$ was used to study mechanically-developed bread doughs made from red spring wheat flour. Doughs were mixed for a fixed time under different headspace pressures to entrain different concentrations of bubbles within the dough. At this frequency, ultrasonic attenuation increased substantially as headspace pressure was increased, while ultrasonic velocity (which is related to the longitudinal modulus of the dough) decreased dramatically. Doughs containing shortening $(0,2$, $4,8 \% \mathrm{w} / \mathrm{w})$ were also prepared, with doughs mixed for various times at atmospheric pressure or under vacuum (0.04 atm). As mixing time increased when mixing at atmospheric pressure, velocity and density decreased while attenuation increased due to air entrainment. Similar trends were observed for doughs prepared with shortening, but velocities and densities at a given mixing time were progressively lower as shortening levels increased. Little variation in velocity or density was observed with mixing time for vacuum-mixed doughs, but changes in attenuation coefficient suggested that ultrasound was sensitive to the changes in dough matrix properties during dough development. Using similar breadmaking doughs, but with either yeast or chemical leavening reagents added, ultrasonic velocity and attenuation were measured as the dough fermented, using a setup in which the dough was allowed to expand freely in a plane perpendicular to the ultrasonic propagation direction. Changes in density of the proving dough was measured using a dynamic dough density system based on digital photography. For vacuum mixed doughs, there was a rapid reduction in velocity in the early stages of fermentation, even though density changed little over the same time period, indicating that fermentation products produced by yeast had a large effect on the properties of the dough matrix. Different chemical leavening systems confirmed that ultrasound was sensitive to effects associated with the dough matrix as well as gas cells since velocities differed at identical void fractions. We conclude that ultrasonic techniques provide a useful tool for studying the effects of bubbles and gas cells in opaque food systems such as dough, and can provide unique insights into events taking place in food processing operations.
\end{abstract}

